le portiQue $\begin{array}{ll}\text { Le Portique } \\ \text { Revue de philosophie et de sciences humaines }\end{array}$

45-46 | 2021

Art et politique, une approche critique

\title{
Penser les répressions politiques avec Varlam Chalamov
}

\section{Konstantin Timachov}

\section{(2) OpenEdition}

1 Journals

\section{Édition électronique}

URL : http://journals.openedition.org/leportique/3733

DOI : $10.4000 /$ leportique.3733

ISSN : $1777-5280$

Éditeur

Association "Les Amis du Portique"

\section{Édition imprimée}

Date de publication : 10 janvier 2021

Pagination : $57-73$

ISBN : 978-2-916332-48-2

ISSN : 1283-8594

\section{Référence électronique}

Konstantin Timachov, "Penser les répressions politiques avec Varlam Chalamov », Le Portique [En ligne], 45-46 | 2021, document 4, mis en ligne le 10 mars 2021, consulté le 26 mars 2021. URL : http:// journals.openedition.org/leportique/3733 ; DOI : https://doi.org/10.4000/leportique.3733

Ce document a été généré automatiquement le 26 mars 2021.

Tous droits réservés 


\title{
Penser les répressions politiques avec Varlam Chalamov
}

\author{
Konstantin Timachov
}

1 Dans cet article, nous abordons l'œuvre littéraire du poète et écrivain russe Varlam Chalamov (1907-1982). Elle nous intéresse surtout vis-à-vis du problème de la connaissance dans l'art. Notre hypothèse consistera à démontrer que son écriture ne se réduit ni à la description des camps, ni à la réminiscence des événements, ni au témoignage. D'abord, nous donnerons quelques informations sur la vie et l'œuvre de Chalamov. Puis, nous analyserons le genre singulier des Récits de la Kolyma et y trouverons deux pôles, otcherk et nouvelle (au sens de Victor Chklovsky), qui se présentent comme deux tendances différentes unies dans les six cycles des récits et aussi, souvent, dans chacun des récits. La première conclusion à laquelle nous arriverons, c'est que certains récits ne peuvent être réduits ni à une source d'information, ni à la narration descriptive, mais qu'ils sont des recherches que l'auteur mène en posant des questions, explicites ou implicites, et dont les réponses se concentrent sur des symboles tels que la brouette. Après, nous démontrerons que dans l'œuvre de Chalamov, il y a une forme de pensée tout à fait spécifique qui est une manière de connaître la réalité vécue aux camps de l'époque stalinienne. Au-delà du témoignage, cette pensée prend une forme de recherche du sens. Nous tracerons ses caractéristiques et analyserons deux récits, La Brouette I et La Brouette II, afin de comprendre les camps dans leurs aspects techniques, économiques et politiques, aspects qui sont au cœur de ces récits. Enfin, nous en viendrons à la deuxième conclusion selon laquelle la distinction par Chalamov des niveaux techniques et « métatechnique » a pour le but de découvrir le sens des camps en saisissant la structure aliénante de l'organisation du travail.

Dans un texte autobiographique intitulé « Ma vie - mes différentes vies », Varlam Chalamov écrit :

«Dans un article on a écrit que j'avais vécu avec notre État tous ses moments cruciaux. C'est une expression exacte. $»^{1}$ 
3 Né à Vologda, l'auteur des célèbres Récits de la Kolyma quitta sa ville natale au NordOuest de la Russie en 1924, l'année de la mort de Lénine, pour travailler et étudier à l'université à Moscou. En 1929, Léon Trotski fut expulsé de l'URSS, Chalamov fut arrêté pour son engagement dans l'opposition anti-stalinienne et passa environ trois ans aux camps de travail dans l'Oural. En 1937, pendant la Grande Terreur, il fut arrêté une seconde fois et passa quatorze années dans les camps de la région de la Kolyma, mais ne put quitter cette région qu'après la mort de Staline en 1953 et ne rentra à la capitale qu'en 1956, après le fameux XX ${ }^{\mathrm{e}}$ congrès du Parti Communiste de l'Union Soviétique qui proclama la déstalinisation.

4 Le deuxième arrêt et la déportation aux camps au Nord-Est de la Russie le privèrent non seulement de sa famille et de ses amis, mais aussi de son métier de journaliste, et cela brisa sa carrière débutante d'écrivain et poète. Composant des vers depuis son enfance, Chalamov recommença déjà à écrire des poèmes dans les camps, ses Cahiers de la Kolyma. Finalement, seule une mince partie de son œuvre a pu voir le jour, ce qui a été suffisant pour qu'après son décès, la nécrologie le nomme « un poète soviétique reconnu », mais il ne s'agit que de la renommée « officielle » d'un membre de l'Union des écrivains soviétiques. Le lectorat à l'étranger - où, en russe ou traduits, ses textes s'infiltrèrent, notamment aux États-Unis, en France et en RDA - et les lecteurs du " samizdat " "l'associèrent surtout à ses fameux Récits de la Kolyma, six cycles en prose qu'il écrivit de 1954 à 1973 et dont un seul récit parut en URSS de son vivant. Ce mode de circulation des textes comme l'intérêt particulier de son lectorat à l'histoire censurée déterminèrent et conditionnèrent la manière de lire les Récits.

\section{Histoire, littérature et recherche dans les Récits de la Kolyma}

5 En effet, après avoir accepté de publier la fameuse nouvelle Une journée d'Ivan Dénissovitch de Alexandre Soljénitsyne, le pouvoir soviétique crut que le thème des camps serait épuisé. Dans un contexte où l'information sur l'époque stalinienne était sous le contrôle de l'État et avant que les historiens et les spécialistes n'aient pu accéder aux archives, des livres autobiographiques comme le roman Le Vertige de Evguénia Guinzbourg ont d'abord été perçus comme une source exclusive d'information sur les camps de l'époque stalinienne.

6 Étant donné leur contenu riche de détails descriptifs, les Récits n'y ont pas fait exception. Par exemple, ils ont été une source importante pour l'auteur de L'Archipel du Goulag notamment, qui y a puisé beaucoup bien qu'à ses propres fins et malgré Chalamov $^{3}$. De la même manière bien que dans une autre situation, l'éditeur émigré aux États-Unis Roman Goul, sans permission de l'auteur et d'une manière scandaleuse mais tout à fait symptomatique, abrégea les Récits en mettant en relief les descriptions des camps $^{4}$. En 1966, dans l'avant-propos des premiers récits publiés dans sa revue Novy journal, Goul les appelle "un document humain d'une valeur exceptionnelle ${ }^{5}$. Yacha Klots montre que, par exemple, Goul a essentiellement révisé le récit Cherry-Brandy, paru à Novy journal en 1968, « de manière à ce que ce récit se lise comme un témoignage documentaire (presque des mémoires) $»^{6}$.

7 Cependant, si tendancieuse et réductrice soit-elle, cette lecture qui ne s'intéresse qu'aux faits n'est pas infondée. Elle pourrait même être sanctionnée par la formule paradoxale de Chalamov : "Non pas la prose des documents, mais la prose éprouvée comme document $»^{7}$. Or, un écrivain fidèle à une telle maxime serait un empiriste radical, il 
n'écrirait qu'à partir de son expérience vécue. C'est pourquoi les historiens des répressions politiques de l'époque du Goulag ont pu parfois y trouver des fils conducteurs dans leurs recherches ${ }^{8}$. La « documentalité » des Récits a été analysée aussi dans plusieurs études, qui essayaient de les classifier au sein de la " littérature des camps ", sous la rubrique de l'« expérience concentrationnaire ". Dans un article, Mireille Berutti indique qu'en général, il y a deux « couches ou groupes » des otcherks ${ }^{9}$ sur le Goulag: "d'une part, l'otcherk-document, le témoignage, de l'autre, l'otcherkrecherche. ${ }^{10}$ Le premier se présente comme un texte d'un témoin, qui décrit son expérience, tandis que le dernier n'est pas obligatoirement écrit à partir du vécu de l'auteur ${ }^{11}$. Cependant, ces deux figures peuvent se réunir chez un même auteur, qui sera non seulement un témoin, mais aussi un "interprète " de son propre témoignage, comme, selon Berutti, dans le cas de Chalamov. D'après Berutti, ces deux couches sont hiérarchisées : tout d'abord, l'auteur écrit un témoignage, puis il y ajoute ses réflexions, comme si l'information factuelle était détachable de la forme d'expression, ainsi que de la narration - de la pensée et son déroulement ${ }^{12}$.

8 Toutefois, les historiens comme Roguinsky, ainsi que les spécialistes contemporains de littérature trouvent que les Récits sont surtout de la littérature. Le philologue de Prague Yan Makhonine pense notamment que " chercher dans la prose de Chalamov une "documentalité" au sens littéral serait fautif et, même s'ils sont intéressants, beaucoup d'efforts d'y tirer des faits et les analyser sous l'angle d'une certitude documentaire ou d'une exactitude historique, à mon avis, contredisent l'intention de leur auteur ${ }^{13}$. À son tour, Éléna Mikhaïlik écrit que «Chalamov n'est pas un rescapé - à ses yeux, il n'est pas un témoin non plus. [...] Il est un écrivain, un artiste, qui rend exploitable un nouveau territoire, qui est hors de la culture - à travers un langage. Et aussi étrange soit-il, ce territoire n'est pas le Goulag en tant que tel $»^{14}$.

D'après elle, selon sa formule précitée, l'épreuve, dont Chalamov fait un point de départ pour toute écriture, est à un tel point déformante que l'auteur ne pourrait pas dire qu'il a survécu : « tous sont morts $»^{15}$.

10 Il est très significatif que Chalamov n'acceptait pas le titre de " chronographe de la Kolyma » et préférait celui de " chronographe de son âme ». En vérité, selon la formule chalamovienne, les Récits sont tout d'abord de la prose - en tant que genre littéraire. Il y a en effet aux yeux de Chalamov une certaine méfiance envers les genres classiques : « Le roman est mort. Et aucune force ne parviendra à ranimer cette forme littéraire. $»^{16}$ Chalamov semble vouloir repartir de zéro et réinventer la littérature, écrire une " nouvelle prose ». Il nous semble que les Récits s'enchaînent dans une longue tradition russe des récits courts d'un genre tout à fait spécifique, celui des otcherk (comptesrendus, essais), un genre auquel appartiennent notamment les trois nouvelles des Récits de Sébastopol (1855-1856) de Léon Tolstoï dans lesquelles il raconte son expérience lors du siège de Sébastopol pendant la guerre de Crimée. Victor Chklovski distingue ainsi ce genre de la nouvelle: « la nouvelle recèle d'habitude une sorte de question qui demande une réponse ", tandis que "l'otcherk ne la suppose pas : je l'ai vu comme ça. L'affaire a été étrangère pour moi - comprenez-la vous-même $\aleph^{17}$. Ainsi, la nouvelle, réaliste ou fantastique, rend possible une recherche littéraire et une narration subjective, tandis que l'otcherk tend à une description objective, journalistique. Nous supposons que les Récits balancent entre ces deux pôles : certains récits tendent à une description exacte et neutre de ce qui se passe dans une réalité vue, tandis que d'autres mènent une sorte de recherche à partir de questions explicites ou implicites posées par l'auteur ${ }^{18}$. C'est 
donc plutôt l'otcherk-document et la nouvelle- recherche qui créent deux pôles de tension au sein de l'ensemble des six cycles, ainsi que dans un récit particulier, et de manière à ce que la nouvelle enveloppe en quelque sorte le "document ", mais ce dernier l'affecte à la manière d'une rétroaction.

Il nous semble que l'œuvre la plus proche des Récits de Chalamov est À la recherche $d u$ temps perdu de Proust, au sens de la lecture qu'en fait Gilles Deleuze : «La Recherche n'est pas simplement un effort de souvenir, une exploration de la mémoire : recherche doit être pris au sens fort, comme dans l'expression "recherche de la vérité" $»^{19}$. Il ne s'agit pas donc d'une narration à partir d'une simple énumération de souvenirs, mais d'une véritable recherche au sens littéral. De la même manière, l'œuvre de Chalamov n'est pas seulement un rappel d'événements historiques, mais aussi son propre dispositif qui rend possible la connaissance des camps, ainsi qu'un travail d'anamnèse à partir de l'expérience vécue.

Même si les Récits ne sont pas du tout un travail d'historien, la chronologie est très importante pour Chalamov, qui a tendance à dater certains événements. La chronologie l'aide non seulement à remettre en ordre ses souvenirs, mais aussi à suivre l'évolution des camps dans leur généalogie. En lisant les Récits, on tombe assez souvent sur des énumérations, des classifications, des typologies, des comparaisons ${ }^{20}$ ou même des « lois " telles que la " loi de la résistance à la décomposition ». Elles se mêlent à des faits racontés de manière à ce que le narrateur se présente comme un véritable penseur qui mène une recherche à partir de son expérience, ce qui se résume dans le texte nommé Ce que j'ai vu et compris au camp (1961) ${ }^{21}$.

13 Assez souvent, Chalamov pose explicitement une question, puis raconte tel ou tel événement. Ce sont souvent des questions qui posent un problème, comme par exemple dans le récit Comment tout a commencé. Cette question y est répétée plusieurs fois, jusqu'à la fin où le narrateur trouve une réponse. Ou encore dans La Brouette I, où l'auteur pose aussi une question explicite : «De l'or, il y en a beaucoup, mais comment le prendre ? $\|^{22}$ Dans ces exemples, chaque question inaugure une recherche, alors que dans d'autres récits, ce sont des questions implicites qu'une phrase recèle et qui pose un problème à résoudre.

Il est possible que le mélange de ces deux genres (compte-rendu et nouvelle) soit un effet, et un révélateur d'une opération fondamentale - le dédoublement. Cette opération caractérise un processus qui affecte les six parties du cycle des Récits, à tous les niveaux de composition. Tout d'abord, il y a une partie qui s'intitule Les Récits de la Kolyma, écrite en 1954-1960, et Le gant, ou Les KR II ${ }^{23}$ dont la plupart datent de 1970-1973. Puis, il y a des récits qui sont liés l'un à l'autre : la narration se multiplie en plusieurs récits qui racontent un événement de deux perspectives différentes (comme dans Le colis et Oraison funèbre). Enfin, le narrateur se présente tantôt comme " je ", tantôt comme « il ", et sous plusieurs noms (Andreïev, Krist, etc.) ${ }^{24}$.

De même, un récit peut fréquemment receler des citations ou des allusions qui renvoient à un ou plusieurs textes, comme par exemple dans le récit Sur parole qui renvoie aux textes de Pouchkine, Gogol et Hugo. Selon Elena Mikhaïlik, " lorsque l'auteur crée des liens intertextuels, il introduit au récit un ensemble de continuums culturels interconnectés, un ensemble libre et variable $»^{25}$. Autrement dit, on peut y voir aussi une manière d'ouvrir l'espace clos de la narration, un point qui perce les limites temporelle et géographique de la situation décrite, de sorte que l'intertexte devient un procédé du dédoublement implicite ${ }^{26}$. 

Gant, ou Les KR II, donnent un exemple particulier du dédoublement. Ce sont des récits tardifs, écrits à l'époque où son style s'est considérablement développé. Igor Soukhikh y voit « un passage de "la nouvelle prose éprouvée comme un document" au document simple, de la nouvelle et de la structure poétique au compte-rendu et à la mémoire, du symbole au sens immédiat ${ }^{27}$. Soukhikh voit cette évolution non seulement dans Le Gant mais aussi dans les récits de la partie La Résurrection du mélèze (1966-1967). Il est vrai que la structure des deux dernières parties est différente de celle des trois premières, plus récentes. Toutefois, Chalamov revient plusieurs fois à des symboles (le graphite, le gant, etc. ${ }^{28}$ ) et des récits comme Le sentier et La Résurrection du mélèze contiennent un très profond symbolisme et des métaphores poétiques. Ainsi y persistent toujours les deux pôles indiqués ci-dessus.

\section{La Kolyma et le Klondike}

Il se peut que $L a$ Brouette I et La Brouette II s'unissent dans un diptyque : l'un est un essai assez traditionnel, alors que l'autre est plutôt une nouvelle avec beaucoup d'informations factuelles et qui se déroule autour de l'image centrale de la brouette ${ }^{29}$. Si l'on compare les structures de ces textes, on trouve qu'elles sont assez semblables. Le premier commence par une comparaison de la région de la Kolyma avec celle du Klondike ; au début du deuxième récit, on trouve une comparaison de la région de la Kolyma avec celle de Sakhaline et de leurs brouettes respectives. Toutefois, au début du deuxième, il y a aussi des descriptions du système bureaucratique des camps de la Kolyma. Les deux récits parlent du système technique de la carrière ; cependant, le deuxième récit est plus riche en détails, avec des descriptions précises des instruments, des professions, etc. Enfin, tous les deux finissent par la description des routes de la Kolyma construites à cette époque-là par les mains des détenus, mais le premier évoque aussi l'industrie de l'or qui existait avant leur arrivée en 1936. Les deux décrivent l'organisation du travail dans le chantier avec des références à l'expérience vécue de l'auteur, mais le deuxième récit contient plus de réminiscences et même un dialogue, ainsi que ses réflexions, la citation d'un journal et un nombre important de chiffres (la capacité des différentes brouettes, les distances du transport des pierres, etc.). Du deuxième récit nous apprenons que Chalamov y travailla d'août 1937 à décembre 1938.

Chalamov revient plusieurs fois à ce qu'il appelle le « vrai secret de la Kolyma ». La Brouette I évoque le Klondike, la rivière canadienne qui donna son nom à la région en Alaska et que Chalamov appelle un "voisin d'outre-mer de la péninsule de la Tchoukotka $\|^{30}$. Il trouve dans ces deux régions leurs points communs : un climat extrêmement rude, un terrain difficile et de fameux et riches gisements aurifères. En effet, à la fin du XIX siècle, le Klondike attira des dizaines de milliers de prospecteurs durant la fameuse ruée vers l'or (décrite notamment dans les romans de Jack London). Chalamov les rapproche comme si elles n'étaient pas situées dans deux continents différents à une distance de presque huit mille kilomètres :

"Faire venir à la Kolyma des millions d'hommes et leur donner du travail en été, c'est

difficile, mais c'est possible. Seulement que vont faire ces gens en hiver? S'enivrer à Dawson ou à Magadane? " ${ }^{31}$

19 Il est bien probable que cet effacement de la distance indique que, selon Chalamov, jusqu'à un certain moment, il n'y avait pas de différence importante entre ces deux régions. Il nous rappelle aussi que jusqu'à la moitié des années 1930, il y avait des 
chercheurs d'or embauchés par l'agence Metprécior ou par d'autres. D'après lui, l'écart historique a eu lieu à un certain moment, en 1936, lorsque dans les gisements un tout autre régime du travail est apparu, celui du travail forcé. Cette transformation s'est opérée non seulement dans l'arrivée des détenus, mais aussi dans la refonte complète du système technique et dans l'introduction d'une nouvelle organisation du travail, qui a changé profondément les relations entre les travailleurs (y compris entre les salariés qui ont continué à y travailler).

Dès lors, la Kolyma s'oppose au Klondike, elle n'est pas le symbole d'un miraculeux pays de l'or et d'un enrichissement rapide, mais plutôt celui d'une douleur permanente et des souffrances physiques : les orpailleurs de l'Amérique du Nord furent animés pas la fièvre, tandis que, comme le dit Chalamov, "la vie réchauffe à peine les hommes moribonds »- la quintessence du Goulag. On peut y voir un double sombre du Klondike, "soviétique ", d'autant plus que le chemin vers l'or n'a pas été creusé par l'aventurisme et le désir de s'enrichir, mais par un programme d'État qui organisa l'industrialisation de la région «à la main, à la pioche et à la brouette $\|^{32}$ par des prisonniers dont des dizaines de milliers ont trouvé la mort ${ }^{33}$.

Le voisinage de la Kolyma et du Klondike, indiqué ci-dessus, se retrouve non seulement dans le texte de Chalamov, mais aussi, sans doute, dans l'imagination des idéologues de l'industrialisation rapide. Ces idéologues inspirèrent les investigations géologiques dans des régions éloignées afin de rechercher des métaux précieux, envoyèrent même des ingénieurs aux États-Unis afin de s'initier aux techniques de prospection et d'extraction, et invitèrent les spécialistes américains. Par ailleurs, Mireille Berutti suppose :

"À la même époque [à la fin de la NEP, à la fin des années 1920] Staline, dit-on, nourrit une soudaine passion et une grande curiosité pour l'aventure américaine de la ruée vers l'or en Californie au siècle précédent. Il avait lu une traduction sur le sujet L'Or de Blaise Cendras et des ouvrages américains. $\|^{34}$

22 N'est-il pas caractéristique que dans le monde des Récits, des livres comme le recueil de poèmes Sur la parole de Victor Hugo ou Le côté de Guermantes de Marcel Proust ne purent être utiles que comme feuilles de papier, matériel de production de cartes à jouer, alors qu'on pouvait pourtant y rencontrer des lecteurs de Jack London?

\section{La Kolyma, Sakhaline et Cayenne}

Dans La Brouette I, Chalamov ne fait que tracer brièvement les aspects de cette transformation tragique (en les développant ensuite, dans La Brouette II). Mais il s'y dégage déjà la problématique de ce que l'on pourrait appeler la centralité du travail, selon l'expression de Christophe Dejours ${ }^{35}$. Il y mentionne notamment des « recettes techniques et méta-techniques pour "boucler" le plan $»^{36}$. Dans cette distinction, on peut identifier respectivement deux niveaux : le niveau technique (les outils, les instruments, etc.) et le niveau de l'organisation $\mathrm{du}$ travail, qui a toutes les caractéristiques de l'organisation scientifique du travail élaborée par Taylor. Le premier est décrit littéralement comme une chaîne dans laquelle chacun des maillons correspond à une étape de travail : « le roulage, la préparation du sol, le dynamitage, le concassage et le transport étaient étroitement coordonnés. ${ }^{37}$ Cette chaîne est parfaitement calculée et rationalisée : "Combien d'hommes faut-il affecter au chargement et combien au roulage ? Deux personnes suffisent-elles pour constituer un "maillon", ou bien en faut-il trois? $»^{38}$ 

des privilèges : les truands. Souvent, ils ont été des chefs de brigade, leurs mines se sont trouvées beaucoup plus près du lieu de dépôt, les trajectoires de leurs brigades ont été ainsi plus courtes, leur productivité était plus haute et ils ont donc pu bénéficier de plus de nourriture. Par contre, les personnes concernées par l'« article $58 »^{43}$, comme le fut par exemple l'auteur des Récits, n'ont fait que des travaux extrêmement pénibles, dans les conditions les plus dures, en conséquence de quoi ils n'ont pu qu'à peine survivre. Dans tout ce système d'exploitation, Chalamov a trouvé " une raison économique, une raison politique et une raison inhumaine meurtrière $»^{44}$. de la Russie monarchique à Sakhaline ${ }^{45}$, dont une description se trouve notamment dans L'île de Sakhaline d'Anton Tchekhov ou dans Sakhaline : le bagne de Vlas Dorochévitch qui contiennent des descriptions de souffrances horribles et qui ont d'ailleurs provoqué des débats publics sur ce sujet. À cette époque-là, les détenus travaillèrent aussi avec des brouettes. Chalamov souligne qu'à Sakhaline, ils étaient attachés aux brouettes, ce qu'il voit comme "plutôt une torture morale " n'était pas le cas de la Kolyma du Goulag où les brouettes étaient plus grandes, ce qui en conséquence, rendait le travail plus pénible. La nécessité d'augmenter leur capacité a été dictée par des fins économiques. Chalamov cite l'article «Le problème des brouettes » publié par le journal La Kolyma soviétique en novembre 1936 qui admet que « le rythme et le coût de production dépendent en grande partie de la fabrication de ces engins ». Juste après cette référence historique, il évoque un camarade qui travailla avec lui au printemps de 1938, « un communiste français Derfel venant des carrières du 
bagne de Cayenne $»^{47}$. Ayant l'expérience du bagne, Derfel a survécu un mois de plus que ses camarades. Sans aucun doute, Chalamov évoque ces bagnes afin de démontrer que le stalinisme n'a pas mis fin aux répressions politiques en Russie, mais que bien au contraire, il les a aggravées de sorte que les vieilles formes des persécutions ont été récupérées et rendues encore plus inhumaines.

Au début du récit, on trouve encore une très grande intuition de Chalamov : " La brouette, la brouette du détenu est le symbole de toute une époque. La machine de l'Osso $^{48}$ / Deux brancards et une roue. $»^{49}$ D'un certain point de vue, ce n'est pas seulement la brouette qui est une machine, mais aussi tout le système, que les hauts responsables avaient conçu afin d'exploiter les détenus, et qui ressemble à une grosse machine. Chalamov explique comment celle-ci fonctionne : dans la Kolyma, la main du destin, ce n'est pas le hasard, mais un dossier personnel qui garde une copie de la décision de l'Osso et ses directives spéciales selon lesquelles le détenu doit être employé uniquement à des travaux physiques pénibles; le détenu est par ailleurs privé du droit de correspondance; les autorités des camps doivent informer l'administration à Moscou une fois tous les six mois.

Entre Moscou et la Kolyma existaient donc des communications, et la distance n'était que géographique : "le plan de l'État pénètre jusque dans le gisement et dans le secteur, jusque dans la brigade et le maillon. $\aleph^{50}$ Chalamov décrit soigneusement toute la hiérarchie administrative qui pèse sur les détenus, sur leur lieu de travail, et qui les surveille : le chef de brigade, le contremaitre, le surveillant, le responsable des travaux, le chef de secteur, le directeur de gisement, mais aussi le soldat d'escorte, l'employé de la section locale du parti affecté au gisement, le délégué régional et son réseau d'informateurs, le représentant de la Direction des camps de Magadane, et enfin le représentant du Goulag venu de Moscou.

Certes, il y avait une opposition forte entre les détenus et l'administration, mais ce que Chalamov souligne constamment, c'est la hiérarchie parmi les détenus. D’après lui, l'administration des camps a réussi à opposer les travailleurs les uns contre les autres. " Ce sont justement tes camarades qui veillent à ce que tu n'escroques pas l'État, à ce que tu ne te reposes pas de façon intempestive. À ce que tu mérites ta ration. ${ }^{51} \mathrm{Si}$ les ordres de Moscou ont pu traverser des milliers de kilomètres, pénétrer le gisement et contrôler chaque habitant des camps de la Kolyma, c'était à cause de l'absence de solidarité parmi eux. Ce fut la tragédie de tout ce monde qui se trouvait aux camps : l'administration a réussi à faire disparaître l'entraide et la solidarité parmi les habitants des camps - la loi du Klondike, chacun pour soi, n'a pu qu'aggraver leurs conditions de vie. La morale des criminels, des truands, qui étaient souvent des chefs de brigade, "meurs, toi, aujourd'hui, et moi, je mourrai demain ", est devenue un principe pour beaucoup de personnes qui étaient loin de leur " monde ». C'est ce qu'Alain Badiou a souligné : "Pour Chalamov, le crime de Staline est moins les camps que d'avoir donné, dans les camps, puissance et latitude aux truands, à raison de ce que, en face d'eux, il n'y a ni conscience collective ni principe fermes. ${ }^{52}$ Cependant, dans ses récits, Chalamov revient plusieurs fois sur les formes de résistance des détenus. Par exemple, dans Triangulation de classe III, il raconte comment 75 trotskistes vivaient dans une baraque et ont collectivement refusé de travailler à l'époque où ils pouvaient faire grève légalement, mais avec le resserrement du régime politique pendant la Grande Terreur, ils ont été exécutés. 
Quant à Chalamov, il n'a pas accepté la morale des truands. Dans le texte Ce que j'ai vu et compris aux camps, il dit : " Je suis fier d'avoir décidé dès le début, en 1937, que je ne serais jamais un chef de brigade si ma volonté pouvait entraîner la mort d'une autre personne - si ma volonté devait servir les autorités en opprimant d'autres prisonniers comme moi. » Il est évident qu'une telle forme de résistance a été à contre-courant et s'est opposée aux principes même de l'organisation du travail à son niveau " métatechnique ».

On voit bien à quel point les Récits sont différents du genre classique du récit : l'épreuve vécue d'un survivant est ce qui anime la recherche du sens des camps. Le procédé du dédoublement, comme nous l'avons montré plus haut, ouvre les récits à un contexte plus large et les fait communiquer avec d'autres événements historiques, tels que l'industrialisation de la région qui visait à transformer la Kolyma en une sorte de Klondike soviétique. Un questionnement à partir de l'idée de la centralité du travail contribue à rechercher le sens de ces camps conçus dans les buts économique et politique. En suivant la pensée de Chalamov, on en vient à la conclusion que les camps de l'époque stalinienne s'enchaînent dans une longue histoire des persécutions politiques du type des bagnes, utilisant le travail forcé pour étouffer l'opposition politique, comme en témoignent les exemples de Derfel ou des trotskistes anonymes.

\section{NOTES}

1. Varlam Chalamov, Euvres en 6 tomes et 1 tome complémentaire, t. IV, Moscou, Éd. Knijni Kloub Knigovek, 2013, p. 297 (en russe).

2. Еn russe САмИздАТ ( officielles du régime soviétique, effectuée par des réseaux qui faisaient circuler des textes censurés ou qui ne pouvaient pas être publiés autrement.

3. En 1968, dans un cahier, Chalamov écrivit : «Par l'intermédiaire de Khrabrovitsky, j'ai informé Soljénitsyne que je n'autorisais l'utilisation d'aucun fait de mon travail pour son travail. S. n'est pas une personne idoine à cela " (https://shalamov.ru/library/23/14.html). Valéry Yéssipov arrive à la conclusion : «La manière, dont Soljénitsyne a traité certains faits, n'a pas toujours été dictée par les principes d'une rédaction purement " artistique », mais par des motifs idéologiques » (Le livre qui a trompé le monde : recueil d'articles critiques et des documents sur L'Archipel du Goulag de A. I. Soljénitsyne, éd. V. V. Yéssipov, Moscou, Éd. Letni sad, 2018, p. 443-444 (en russe)).

4. Yacha Klots, «Chalamov vu par l'émigration russe. Les Récits de la Kolyma dans le Novy journal » in « La loi de la résistance à la décomposition... » Caractéristiques de la prose et de la poésie de Varlam Chalamov et leur perception au début du XXI siècle, éd. Loukach Babka, Sergueï Soloviev, Valéry Yessipov, Jan Makhonine, Prague- Moscou, 2017, p. 231-257 (en russe). D’autres exemples in Eléna Mikhaïlik, Comète illégale. Varlam Chalamov : essai d'une lecture lente, Moscou, Éd. Novoïé litératournoïe obozrénié, 2018, p. 11-12 (en russe).

5. Le slaviste Clarence Brown, qui emporta un manuscrit des Récits de Moscou à New York, écrivit à Goul : «ils [les Récits] pourraient être d'une importance explosive » (may prove to be of explosive importance). Cette citation est tirée de Yacha Klots, « Varlam Shalamov between Tamizdat and the Soviet Writers' Union (1966-1978) », in Russian Literature, Vol. 96-98, February-May 2018, P. 
137-166, précédemment publié en russe in Colta, le 10 janvier 2017, https://www.colta.ru/ articles/literature/13546-varlam-shalamov-mezhdu-tamizdatom-i-soyuzom-sovetskihpisateley-1966-1978.

6. Idem. Il est symptomatique aussi qu'après s'être infiltré à l'étranger en 1966, les Récits parurent dans des revues en trois-quatre récits par numéro et ne furent publiés en russe dans un livre qu'en 1978 à Londres.

7. En russe : « НЕ ПРОЗА ДОКУМЕНТОВ, А ПРОЗА ВЫСТРАДАННАЯ КАК ДОКУМЕНТ » (Varlam Chalamov, Euvres en 6 tomes et 1 tome complémentaire, t. V, Moscou, Éd. Knijni Kloub Knigovek, 2013, p. 157). Cette maxime est tirée d'un texte posthume qu'on appelle Sur la "nouvelle prose ", de 1965. Dans ce manifeste, Chalamov explique son programme éthique et esthétique en faisant référence à ses "prédécesseurs ", Melville et Saint-Exupéry. On trouve déjà des réflexions semblables dans le récit La cravate de 1960, puis dans Le gant de 1972.

8. Arséniï Roguinsky, "Du témoignage à la littéraire ", in Varlam Chalamov dans la littérature et l'histoire soviétique, ed. S. M. Soloviev, Moscou, Éd. Litéra, 2013, p. 12-14 (en russe), https:// shalamov.ru/research/295.

9. En russe, compte-rendu littéraire ou essai documentaire. Ci-dessous, ce genre est abordé à partir de la théorie de Viktor Chklovski.

10. Mireille Berutti, "Varlam Chalamov : littérature comme document " in Centenaire de la naissance de Varlam Chalamov, Moscou, Éd. DerDieDasGroupp, 2007, p. 199-208 (en russe), https:// shalamov.ru/research/99.

11. Sans aucun doute, c'est le cas de L'Archipel du Goulag (et d'un certain point de vue de Une journée d'Ivan Dénissovitch aussi), dont l'auteur, qui avait une connaissance des camps très limitée, a tiré l'information reçue de 227 personnes, selon la Préface, et l'a interprétée dans une forme d'" essai d'investigation littéraire ".

12. D'après elle, ceci est vrai pour les récits de Chalamov, en particulier pour les derniers. Cf. Ibid., p. 199-208 (en russe).

13. Yan Makhonine, "Documentalité de la prose de Chalamov comme une stratégie littéraire » in «La loi de la résistance à la décomposition... » Caractéristiques de la prose et de la poésie de Varlam Chalamov et leur perception au début du XXI siècle, op. cit., p. 123 (en russe).

14. Eléna Mikhaïlik, Comète illégale. Varlam Chalamov : essai d'une lecture lente, op. cit., p. 15 (en russe).

15. Ibid., p. 11. Cf. «Pluton remontant de l'enfer plutôt qu'Orphée descendant en enfer » (Varlam Chalamov, «De la prose ", in Varlam Chalamov, Euvres en 6 tomes et 1 tome complémentaire, t. V, op. cit., p. 151).

16. «Il n'y a pas de mémoires dans Les Récits de la Kolyma. Je n'écris pas non plus des nouvelles, ou bien je n'essaye pas d'écrire une nouvelle mais ce qui ne serait pas de la littérature » (Ibid., p. 157).

17. Victor Chklovski, L'énergie de l'erreur : Livre sur sujet, Moscou, Éd. Sovetsky pissatel, 1981, p. 130-133 (en russe).

18. Dans tout le cycle des récits, Igor Soukhikh compte plus d'une trentaine de récits du genre otcherk. Igor Soukhikh, "Vivre après la Kolyma " in Zvezda, n6, 2001 (en russe), https:// magazines.gorky.media/zvezda/2001/6/zhit-posle-kolymy.html.

19. Gilles Deleuze, Marcel Proust et les signes, Paris, PUF, 1964, p. 7.

20. « Il y a deux types de crimes... », « Le plus terrible, c'était... », « J'ai compris que... », « La conclusion de mon expérience... », etc.

21. Varlam Chalamov, Euvres en 6 tomes et 1 tome complémentaire, t. IV, op. cit., p. 625-627.

22. Varlam Chalamov, Les Récits de la Kolyma, traduit du russe par Sophie Benech, Catherine Fournier, Luba Jurgenson, Paris, Éd. Verdier, 2003, p. 1319.

23. En russe, « KR » est une abréviation composée des initiales du titre « Récits de la Kolyma». 
24. Cf. Mikhaïl Mikheïev, Narrateurs multiples dans les récits de Varlam Chalamov, en ligne, https:// shalamov.ru/research/173 (en russe).

25. Eléna Mikhailik, op. cit., p. 61 (en russe).

26. Il y a même des récits comme L'écureuil ou La croix qui se déroulent loin de la Kolyma, à Vologda, la ville natale de Chalamov.

27. Igor Soukhikh, op. cit.

28. Par exemple, le graphite, « le frère du diamant », est un symbole d'immortalité, et les notes qu'un topographe fait sur les tronques des mélèzes sont des inscriptions "presque éternelles ». Comme pour Chalamov le mélèze symbolise un être humain, on peut interpréter l'inscription sur un mélèze faite par un crayon de graphite comme une métaphore du mécanisme de mémoire qui garde les souvenirs de la Kolyma.

29. Igor Soukhikh pense que La Brouette I, ainsi que certains autres récits dans le cycle Le Gant, serait inachevé, étant assez court, deux pages environ. Cf. Igor Soukhikh, Ibid. En vérité, il y a plusieurs récits de même taille ou moins longs.

30. Varlam Chalamov, Les Récits de la Kolyma, op. cit., p. 1320.

31. Ibid., p. 1319.

32. Idem.

33. L'industrie aurifère joue, jusqu'à aujourd'hui, un rôle important en Russie depuis des siècles ; par exemple, en 1913, la réserve d'or de la Banque d'État Russe était la plus grande du monde 1300 tonnes. La production de l'or y fut en baisse pendant la Grande Guerre et la Révolution Russe. Son évolution dans la deuxième moitié des années 1930, présentée par Chalamov, se justifie d'après les historiens qui montrent un renforcement brusque de l'extraction de l'or depuis les années 1920. Contrairement à des mythes répandus autour de l'histoire de cette industrie durant le régime soviétique, les premiers pas vers sa reconstruction ont été faits par les orpailleurs indépendants sous la NEP, encouragés par le décret du Conseil des Commissaires du peuple de 31 octobre 1921 « Sur l'industrie de l'or et du platine » qui propose « d'accorder à tous les citoyens de la RSFSR, coopératives, artels et autres associations le droit d'effectuer des recherches, de l'exploration et de l'extraction de l'or et du platine sur les terres de la RSFSR, et également de recevoir des entreprises et des mines de platine et or de l'État pour une exploitation contractuelle... ", in M. M. Maksimov, Essai sur l'or, Moscou, Éd. Nedra, 1977 (en russe).

34. Mireille Berutti, Varlam Chalamov : chroniqueur du Goulag et poète de la Kolyma, Paris, Éd. de BoD, 2013, p. 203, https://issuu.com/chalamov/docs/chalamov_berutti_p_1-288.

35. Il est bien possible de retrouver dans les Récits toutes les pathologies que ce chercheurmédecin énumère dans son récent article: les pathologies de surcharge, les pathologies consécutives aux violences, les pathologies du harcèlement, les pathologies dépressives, jusqu'au suicide. $C f$. Christophe Dejours et Antoine Duarte, « La souffrance au travail : révélateur des transformations de la société française » in Modern \& Contemporary France, n²6-3, 2018, p. 236. Si nous tirons ce constat d'un tout autre contexte, celui de la France de nos jours, ce n'est pas dans le but d'assimiler deux phénomènes différents, mais afin de souligner l'importance d'attaquer le mal à la racine, ainsi que les conséquences graves que certains types d'organisation du travail entraînent.

36. In Varlam Chalamov, Les Récits de la Kolyma, op. cit., p. 1320, le mot « СВЕРХТЕХНИчЕСКИЕ » est traduit comme « ultra-techniques », mais nous préférons le traduire avec le préfixe méta, au sens de « au-delà ».

37. Ibid., p. 1319.

38. Ibid., p. 1320.

39. Ibid., p. 1319.

40. Christophe Dejours et Antoine Duarte, op. cit., p. 239-240.

41. Varlam Chalamov, Les Récits de la Kolyma, op. cit., p. 1319. 
42. Ibid., p. 1320.

43. Crimes politiques selon le code pénal.

44. Ibid., p. 1333. $C f$. « L'organisation du travail apparaît en fin de compte comme un problème politique » (Christophe Dejours et Antoine Duarte, op. cit., p. 240).

45. Île russe située dans le nord-ouest de l'océan Pacifique, entre la Mer d'okhotsk et la Mer du Japon.

46. Varlam Chalamov, Les Récits de la Kolyma, op.cit., p. 1323.

47. Idem.

48. L'Osso désigne la Conférence spéciale qui à l'époque dépendait du chef de la police d'État (Oguépéou) et qui s'occupa notamment des « ressources humaines » aux camps en y envoyant les détenus.

49. Ibid., p. 1322.

50. Ibid., p. 1324

51. Ibid., p. 1328-1329.

52. Alain Badiou, Peut-on penser la politique?, Paris, Éd. du Seuil, 1985, p. 37.

\section{RÉSUMÉS}

Konstantin Timachov montre comment la prose de Chalamov apporte un éclairage sur un événement historique - les travaux forcés dans les camps de travail à l'époque stalinienne - en inventant une forme d'écriture singulière, à mi-chemin entre un témoignage ou récit historique et une recherche au sens proustien du terme.

Konstantin Timashov shows how Shalamov's prose enlighten us about an historical event -forced labour in the labour camps during the Stalinist era - by inventing a singular form of writing, halfway between a narrative or historical testimony and a research in the way that Proust did.

\section{AUTEUR}

\section{KONSTANTIN TIMACHOV}

Konstantin Timachov est chercheur et enseignant en langues à l'Université nationale de recherche, l'École des Hautes Études en Sciences Économiques, doctorant de philosophie à l'Université Pédagogique Herzen de Saint-Pétersbourg. Ses recherches philosophiques sont élaborées à travers le cinéma, les penseurs et poètes russes et français. 